\title{
Effect of tungsten disulfide nanotubes on crystallization of polylactide under uniaxial deformation and annealing
}

\author{
Fausta Loffredo ${ }^{1 *}$, Loredana Tammaro ${ }^{1}$, Tiziana Di Luccio ${ }^{1,2^{*}} \mathbb{D}$, Carmela Borriello ${ }^{1}$, Fulvia Villani ${ }^{1}$, Saverio De Vito ${ }^{3}$, \\ Karthik Ramachandran ${ }^{2}$ and Julia A. Kornfield ${ }^{2}$
}

\begin{abstract}
Tungsten disulfide $\left(\mathrm{WS}_{2}\right)$ nanotubes (NTs) are examined here as a filler for polylactide (PLA) for their ability to accelerate PLA crystallization and for their promising biocompatibility in relevant to biomedical applications of PLA$W_{2}$ nanocomposites. In this work, we have studied the structural and thermal properties of PLA-WS 2 nanocomposite films varying the concentration of WS 2 NTs from 0 (neat PLA) to $0.6 \mathrm{wt} \%$. The films were uniaxially drawn at $90^{\circ} \mathrm{C}$ and annealed at the same temperature for 3 and 10 min. Using wide angle $x$-ray scattering, Raman spectroscopy and differential scanning calorimetry, we probed the effects of $\mathrm{WS}_{2} \mathrm{NT}$ addition on the structure of the PLA films at various stages of processing (unstretched, stretching, annealing). We found that $0.6 \mathrm{wt} \%$ of WS induces the same level of crystallinity in as stretched PLA-WS 2 as annealing in neat PLA for 10 min. These data provide useful insights into the role of WS 2 NTs on the structural evolution of PLA-WS 2 composites under uniaxial deformation, and extend their applicability to situations where fine tuning of PLA crystallinity is desirable.
\end{abstract}

Keywords: Poly(lactic acid) (PLA), Tungsten disulfide $\left(W_{2}\right.$ ) nanotubes, Nanocomposites, Strain-induced crystallization, Uniaxial deformation, Raman spectroscopy

\section{Introduction}

Polylactic acid or polylactide (PLA) is a polymorphic semicrystalline polymer synthesized from renewable sources that finds applications in diverse fields (food packaging, biomedical devices, drug delivery systems) due its properties of biocompatibility and biodegradability [1]. PLA biocompatibility derives from the fact that in physiological conditions, it degrades by hydrolysis into non-toxic lactic acid that is further metabolized by the human body into carbon dioxide and water as final products. As a result, PLA is widely used as a material for sutures, tissue scaffolds and supports [2-5]. Unfortunately, the applications of this polymer are often limited by its slow crystallization rate, low crystalline degree and

\footnotetext{
* Correspondence: fausta.loffredo@enea.it; tidilu@caltech.edu

'Division for Sustainable Materials, ENEA - Italian National Agency for New Technologies, Energy and Sustainable Economic Development, Piazzale E. Fermi 1, 80055 Portici, NA, Italy

Full list of author information is available at the end of the article
}

low fracture toughness [6-8]. One approach to mitigating these issues is the incorporation of organic or inorganic fillers in the PLA matrix, which can increase the rate of crystallization of PLA and enhance its mechanical properties [9-16]. However, the biocompatibility of the filler and minimal loading concentrations with respect to the polymer matrix are essential requirements from a toxicological point of view, particularly in biomedical applications. In this framework, nanotubes (NTs) of tungsten disulfide $\left(\mathrm{WS}_{2}\right)$ are promising nanofillers for polymer matrices: their intrinsic superior mechanical properties ( $150 \mathrm{GPa}$ Young's modulus, $16 \mathrm{GPa}$ strength, $\sim 14 \%$ elongation [17]) can reinforce polymers, especially under flow-induced crystallization due to their high aspect ratio (diameter $\sim 80-100 \mathrm{~nm}$, length $\sim 2-3 \mu \mathrm{m}$ [18]). They are also reported to possess lower in-vitro and in-vivo cytotoxicity compared to other nanoparticles such as silica or carbon black [18-20]. 
Despite these advantages, to the best of our knowledge, only a few studies are reported in literature on PLA-WS $\mathrm{W}_{2}$ nanocomposites, mainly focused on thermal properties of samples obtained by melt-mixing [2125]. These studies show that $\mathrm{WS}_{2}$ NTs remarkably influence the kinetics of nucleation and growth of the PLA during the cold crystallization. The mechanical properties of solvent cast PLA- $\mathrm{WS}_{2}$ films measured at room temperature have been recently reported by Shalom et al. showing a large increase of elastic modulus ( $50 \%$ for $0.7 \mathrm{wt} \%$ ), yield strength (about $70 \%$ for $0.7 \mathrm{wt} \%$ ), and elongation (about $70 \%$ for $0.5 \mathrm{wt} \%$ ) with increasing concentration of $\mathrm{WS}_{2}$ NTs [24]. On the other hand only our previous work [25] reports on the crystallization process induced by stretching PLA-WS ${ }_{2}$ films at temperatures above the glass transition of PLA $\left(\mathrm{T}_{\mathrm{g}} \sim 55-65^{\circ} \mathrm{C}\right)$ but the effect of the $\mathrm{WS}_{2}$ concentration on the crystallization of PLA was not analyzed at the time.

Therefore, in this work we have prepared PLA-WS films with different amounts of $\mathrm{WS}_{2}(0-0.6 \mathrm{wt} \%)$ with respect to PLA and we have studied the effect of $\mathrm{WS}_{2}$ concentration on the strain-induced crystallization of PLA as a consequence of uniaxial stretching at $90^{\circ} \mathrm{C}$ and constant strain rate. We have also investigated the effect of post-stretching thermal annealing on the crystallization of PLA.

\section{Experimental}

\section{Materials}

All the materials used in this study are commercially available. PLA (Ingeo 4032D, 1.2-1.6\% D-isomer lactide) pellets were purchased from NatureWorks LLC (Minnetonka, MN). Tungsten disulfide nanotubes $\left(\mathrm{WS}_{2} \mathrm{NTs}\right)$ were purchased from Nanomaterials Ltd. (Israel) with diameter of about $80-100 \mathrm{~nm}$ and length of about 2$3 \mu \mathrm{m}$ and they were used as received without any chemical modification. Chloroform was supplied by SigmaAldrich.

\section{Preparation of unstretched and stretched films of PLA and PLA-WS ${ }_{2}$ composites}

Figure 1 reports a scheme of the preparation process of unstretched and stretched films of neat PLA and PLA$\mathrm{WS}_{2}$ nanocomposites with different amounts of $\mathrm{WS}_{2}$ NTs $(0.1,0.2,0.3$, and $0.6 \%$ by weight). The PLA pellets were dried under vacuum at $80^{\circ} \mathrm{C}$ for $4 \mathrm{~h}$ and then at $40^{\circ} \mathrm{C}$ for 3 days to remove moisture prior to the nanocomposite preparation. Suspensions containing the different amounts of $\mathrm{WS}_{2}$ NTs were prepared in chloroform, sonicated for $30 \mathrm{~min}$ and then added to a PLA solution in chloroform $(120 \mathrm{mg} / \mathrm{mL})$ previously prepared by stirring at $40{ }^{\circ} \mathrm{C}$ for about $3 \mathrm{~h}$. The final PLA$\mathrm{WS}_{2}$ suspensions were stirred for $1 \mathrm{~h}$ (Fig. 1a). The PLA$\mathrm{WS}_{2}$ nanocomposite suspensions and neat PLA solution were poured into petri dishes of $\sim 10 \mathrm{~cm}$ diameter (Fig. $1 \mathrm{~b})$ and left at room temperature for 2 days to allow the majority of the solvent to evaporate. After 2 days, the films were dried under vacuum until the solvent was completely removed (no change in mass detected).

All the solvent cast films were hot pressed into $100 \mu \mathrm{m}$ thick films by a Carver laboratory press at $180^{\circ} \mathrm{C}$ for $10 \mathrm{~min}$ and rapidly quenched into an icewater bath to avoid crystallization (Fig. 1c). The weight average molecular weight $\left(\mathrm{M}_{\mathrm{w}}\right)$ of the hot-pressed films (hereafter referred to as UNST) of PLA and PLA-WS nanocomposites was comparable to that of the original PLA pellets $\left(\mathrm{M}_{\mathrm{w}} \sim 100 \mathrm{Kg} / \mathrm{mol}\right)$ as measured by gel permeation chromatography.

Strips cut from the hot-pressed PLA and PLA-WS films were stretched in a dynamometric apparatus at a temperature of $90^{\circ} \mathrm{C}$. After stretching, some films were immediately removed from the instrument (samples called ST0 in Fig. 1d) while another set of films (with the same composition of the ST0 series) was kept at constant length in the stretching apparatus and thermally annealed at $90^{\circ} \mathrm{C}$ for 3 or 10 min (samples ST3 and ST10, Fig. 1e). All the stretched films (ST0, ST3 and ST10) were quenched in ice-water after removal from the instrument.

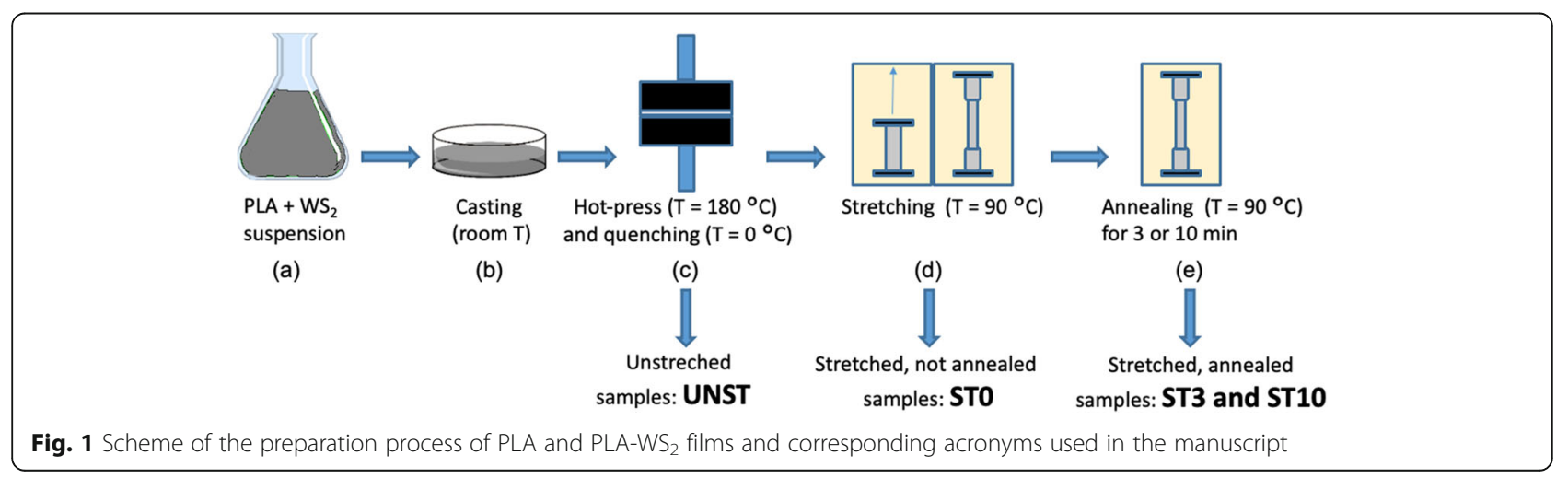




\section{Methods of film characterization}

The dispersion of the $\mathrm{WS}_{2}$ NTs in UNST PLA-WS films was studied by using a Zeiss Axio scope A1 microscope in transmission and bright field mode.

The mechanical properties of the films were measured by uniaxial stretching experiments using an INSTRON dynamometric apparatus (mod. 4301) with 1 kN-load cell. Rectangular sections (width $=5 \mathrm{~mm}$, length $\sim 50$ $\mathrm{mm}$ ) were cut from the hot-pressed films of each composition and mounted in the INSTRON apparatus kept at a temperature of $90{ }^{\circ} \mathrm{C}$. The films were drawn at a strain rate of $500 \mathrm{~mm} / \mathrm{min}$ up to $100 \%$ deformation starting from an initial gauge length of $25 \mathrm{~mm}$. Stressstrain curves were extracted from the forcedisplacement data and the elastic modulus was evaluated from the linear part of the stress-strain curves at deformation of $0.1 \%$ by averaging over ten replicas for each composition.

The structure and crystallinity of as-cast, hot pressed (UNST) and stretched (ST0, ST3, ST10) PLA and PLA$\mathrm{WS}_{2}$ films were probed measuring wide angle $\mathrm{x}$-ray scattering (WAXS) at beamline 5-ID-D of the Advanced Photon Source (APS), Argonne National Labs. The incident X-ray beam with spot-size $250 \mu \mathrm{m} \times 250 \mu \mathrm{m}$ was aligned perpendicular to the film surface. WAXS patterns were acquired on a Rayonix CCD detector that was located $200.83 \mathrm{~mm}$ from the sample. Six patterns were acquired at six different positions along the long side of each film (corresponding to the drawing direction for ST0, ST3, ST10, as an example, see inset in Fig. S2c, showing the position of the six acquisitions for the PLA$\mathrm{WS}_{2} 0.2 \mathrm{wt} \%$ film) with an exposure time of $0.5 \mathrm{~s}$ using X-rays of wavelength $0.7293 \AA$. The six patterns were then averaged in one single scan per sample. Several replicas (3 or 4 films prepared with the same composition, drawing and annealing conditions) were measured for the stretched films (PLA ST0, ST3 and ST10 in Fig. S3, respectively; and the equivalent for the PLA- $\mathrm{WS}_{2} 0.1-$ 0.6 wt\% films in Fig. S4, S5, S6, S7). The scattered intensity per each pattern is reported on an absolute scale after taking into account the calibration factor provided by the beamline, the transmitted counts on the beamstop, the thickness of the sample and the background scattering from air. The $2 \mathrm{D}$ patterns were then integrated into $1 \mathrm{D}$ curves of intensity vs. the scattering vector $q$.

Raman spectroscopy was performed through a Renishaw InVia Reflex Raman spectrometer selecting the laser wavelengths of $514.5 \mathrm{~nm}$ (laser power of 50\%) and using an objective of $50 x$ of magnification. The range of investigated wavelength was $100-1800 \mathrm{~cm}^{-1}$. Each measurement is reported as an average of 20 subsequential accumulations with an exposure time of $10 \mathrm{~s}$. The position, intensity and full width at half maximum
(FWHM) of the Raman bands were evaluated from a Lorentzian fit of the spectra needed to resolve overlapping peaks (see Fig. S11a). The intensity and FWHM values of the bands were then normalized with respect to the PLA band centered at $1095 \mathrm{~cm}^{-1}$ (associated to stretching OCO group [26]) in order to minimize the errors due to the different thickness of the samples. This band was chosen as internal reference because its intensity does not seem to change appreciably from one sample to the other independently on the crystallinity evaluated from WAXS, Raman and DSC analyses. For each type of sample three replicas per condition (composition, drawing, annealing time) were analyzed in their central region and the reported values of normalized intensity and FWHM of the investigated bands are the average of the measurements (obtained after fitting and normalization) made on the three replicas. The error bars correspond to the standard deviation.

The thermal properties were measured by Differential Scanning Calorimetry (DSC) carried out with a Perkin Elmer DSC7 instrument. About $10 \mathrm{mg}$ of sample was placed in an aluminum pan under nitrogen flux and initially held at $30^{\circ} \mathrm{C}$ for $5 \mathrm{~min}$, then heated up to $220^{\circ} \mathrm{C}$ with a rate of $5^{\circ} \mathrm{C} / \mathrm{min}$, held at $225^{\circ} \mathrm{C}$ for to $5 \mathrm{~min}$ and then cooled down to $30^{\circ} \mathrm{C}$ with a rate of $5{ }^{\circ} \mathrm{C} / \mathrm{min}$. In order to investigate the effect of the stretching and annealing on the crystallinity of the samples, the first heating scan was analyzed. In detail, the cold crystallization temperature $\left(T_{c c}\right)$ and the melting temperature $\left(T_{m}\right)$ were determined from the minimum of the crystallization exotherm and from the maximum of the melting endotherm, respectively. The enthalpies of the cold crystallization $\left(\Delta H_{c c}\right)$ and melting $\left(\Delta H_{m}\right)$ were determined by integrating the areas under the corresponding peaks and used to estimate the percentage of crystallinity $\left(X_{c}\right)$ existing before the DSC measurement and not induced by the latter. $X_{c}$ was calculated from the heating curves according to the eq. (1):

$$
X c=\frac{\left(\Delta H_{m}-\Delta H_{c c}\right)}{(1-w t \%) \Delta H_{m}^{0}} * 100
$$

where $\Delta H_{m}^{\circ}$ is the melt enthalpy of theoretically $100 \%$ crystalline PLA (93 J/g, [27]) and wt\% indicates the weight fraction of $\mathrm{WS}_{2}$ NTs in the nanocomposites.

\section{Results and discussion}

The UNST PLA-WS $\mathrm{W}_{2}$ nanocomposite films show a uniform dispersion of $\mathrm{WS}_{2}$ NTs in PLA both at the lowest (0.1\%, Fig. 2a) and highest (0.6\%, Fig. $2 \mathrm{~b})$ concentration of NTs. The optical micrographs in Fig. 2 do not reveal presence of aggregates demonstrating that the preliminary sonication is efficient in unraveling the nanotubes 
and that they readily disperse in PLA without need of further functionalization [21].

The results of the uniaxial stretching experiments of PLA and PLA-WS $S_{2}$ nanocomposites performed in the Instron chamber held at the temperature of $90^{\circ} \mathrm{C}$ are reported in Fig. 3. The stress-strain curves, recorded up to $100 \%$ strain, show a very similar behaviour for the PLA$\mathrm{WS}_{2}$ nanocomposites and neat PLA (Fig. 3a) for all the $\mathrm{WS}_{2}$ concentrations examined here $(0.1-0.6 \mathrm{wt} \%)$. A slight increase of $\sim 10 \%$ of the elastic modulus (from 3 to $3.5 \mathrm{MPa}$ ) is found for the films at $\mathrm{WS}_{2}$ concentrations higher than $0.2 \%$ (Fig. 3b). The error bar length attributed to the modulus (corresponding to the standard deviation) indicates a good reproducibility between the specimens of the same type, which, in the case of the nanocomposites, also confirms the uniform dispersion of the $\mathrm{WS}_{2}$ NTs in the PLA matrix (Fig. 2). The value of the elastic moduli of the films drawn at $\mathrm{T}=90^{\circ} \mathrm{C}$ is lower than PLA samples drawn at room temperature (Fig. S1a-c) by $2-3$ orders of magnitude ( $3 \mathrm{MPa}$ vs. 1.5 GPa, respectively). According to literature [28-32], at drawing temperatures above the glass transition the tensile strength and the elastic modulus of the PLA decrease, whereas the elongation at break significantly increases.

Wide angle $\mathrm{x}$-ray scattering (WAXS) was used to detect variations in the crystallization status of the PLA$\mathrm{WS}_{2}$ nanocomposites after the different steps of preparation. The 2D and 1D diffraction patterns of the as-cast films of PLA and PLA-WS $\mathrm{W}_{2}$ at all concentrations of nanotubes show all reflections of the crystalline $\alpha$-phase of PLA (Fig. S2) [33]. The diffracted intensity is uniformly distributed along rings (black trace, Fig. S2a-e), showing isotropic, crystalline morphology created upon quiescent crystallization conditions of solvent-casting procedure. The Bragg peaks of the $\mathrm{WS}_{2}$ nanotubes are faint in PLA-WS $S_{2}$ spectra at $0.1 \mathrm{wt} \%$ (Fig. S2b) but become clearly visible at $0.2 \mathrm{wt} \%$ (Fig. S2c). The hot press step (where the as-cast films were held at $180^{\circ} \mathrm{C}$ for 10 $\mathrm{min}$ ) and subsequent fast quenching completely make the PLA matrix amorphous (red trace, Fig. S2a-e).
Indeed, only a broad diffuse scattering halo from PLA and the $\mathrm{WS}_{2}$ reflections characterize the WAXS patterns of the UNST films (Fig. S2). The stretching and the subsequent annealing process induced a further change of the PLA structure from the amorphous UNST films to the stretched films ST0, ST3 and ST10 (Fig. 4 and Fig. S8, S9, S10) characterized by a progressive reduction of the PLA amorphous component and corresponding increase of the $\alpha$ crystalline phase. Moreover, the intensity spots in the diffraction 2D patterns of the stretched PLA indicate that the polymer chains are aligned along the stretching direction $x$, drawn in the 2D pattern of PLA ST10 film (Fig. 4a). The same effect of the stretching and annealing was observed for all the nanocomposites with $0.1-0.6$ wt\% (see Fig. $4 \mathrm{~b}$ and Fig. S8, S9, S10). In both cases of PLA (Fig. 4a) and the PLA-WS 2 nanocomposites all concentrations (Fig. $4 \mathrm{~b}$ and Fig. S8, S9, S10) increasing the annealing time from 0 (ST0) to $10 \mathrm{~min}$ (ST10) induces an increase of intensity of the PLA peaks at expense of the amorphous content. To give a quantitative estimation of the crystallinity in Fig. 5 we have plotted the ratio $I_{\text {peak }} / I_{\text {valley }}$ between the intensity value of the main PLA reflection $((110) /(200)$, positioned at $q=1.16 \AA^{-1}$ ) and the intensity value at the valley between this and the (203) peak (positioned at $q=1.25 \AA^{-1}$ ) as a function of the $\mathrm{WS}_{2}$ concentration (for all patterns shown in Fig. 4 and Fig. S8, S9, S10). The intensity ratio $I_{\text {peak }} / I_{\text {valley }}$ is constant for the amorphous UNST films, but after stretching (ST0) it increases with the concentration of nanotubes first slowly up to $0.2 \mathrm{wt} \%$ and then with a sudden jump at $0.3 \mathrm{wt} \%$, remaining almost constant the final concentration of $0.6 \mathrm{wt} \%$. While the stretching itself does not induce noticeable crystallization in PLA (ST0, $\mathrm{WS}_{2}=$ $0 \mathrm{wt} \%$ ) (but probably just a chain alignment), the nanotubes promote crystallization by acting as nucleation agents [23]. The value of $\mathrm{I}_{\text {peak }} / \mathrm{I}_{\text {valley }}$ for the conditions ST3 and ST10 is very similar to and larger than the corresponding ST0 value. This indicates that the crystallization of PLA has reached its maximum already after 3 min of annealing.
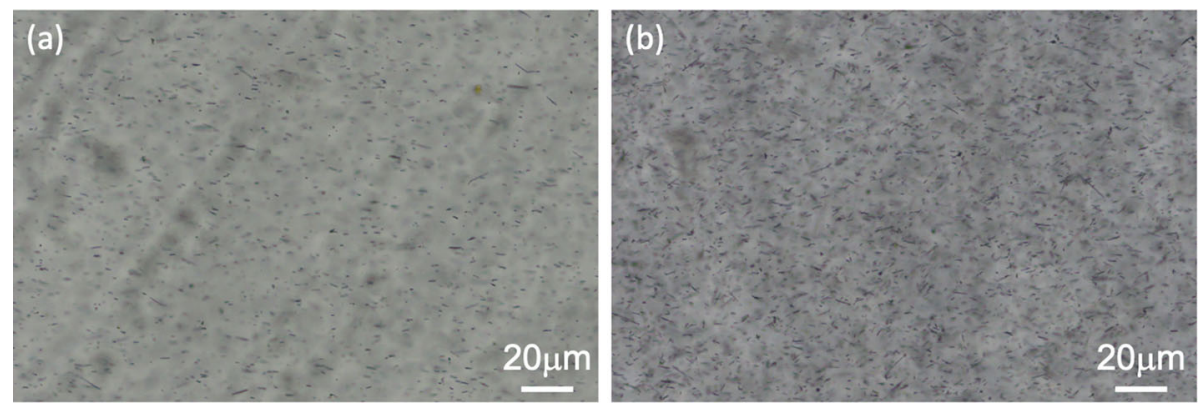

Fig. 2 Optical micrographs of UNST PLA-WS 2 nanocomposite films containing $0.1 \%$ (a) and $0.6 \%$ (b) of WS $\mathrm{WT}_{2}$ NTs 

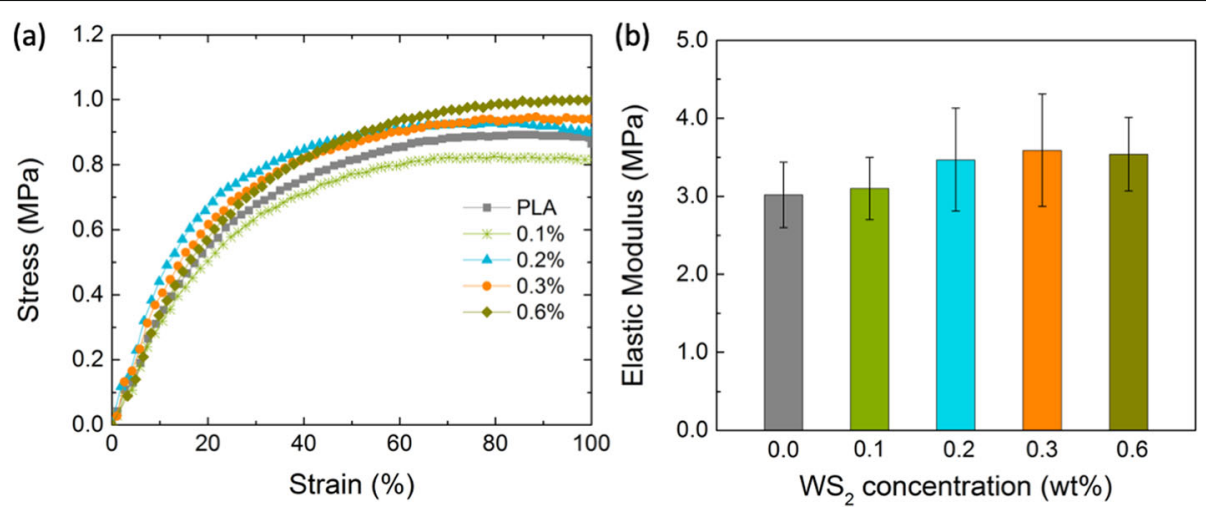

Fig. 3 Stress-strain curves (average of ten repeats) up to $100 \%$ strain, recorded upon drawing the PLA and PLA-WS films at T $=90^{\circ} \mathrm{C}$ with a stretching rate of $500 \mathrm{~mm} / \mathrm{min}$ (a) and calculated elastic moduli (b)

In order to investigate the conformational changes due to the extensional stress and subsequent annealing process, all samples were characterized by Raman spectroscopy selecting the central region of each specimen where, in the stretched films, the mechanical stress should be maximum. The Raman spectra of PLA and PLA-WS ${ }_{2}$ 0.6\% samples (UNST, ST0, ST3, ST10) normalized with respect to the band at $1095 \mathrm{~cm}^{-1}$ (as described in the experimental section) are reported in Fig. 6. The first part of the spectra in the wavelength
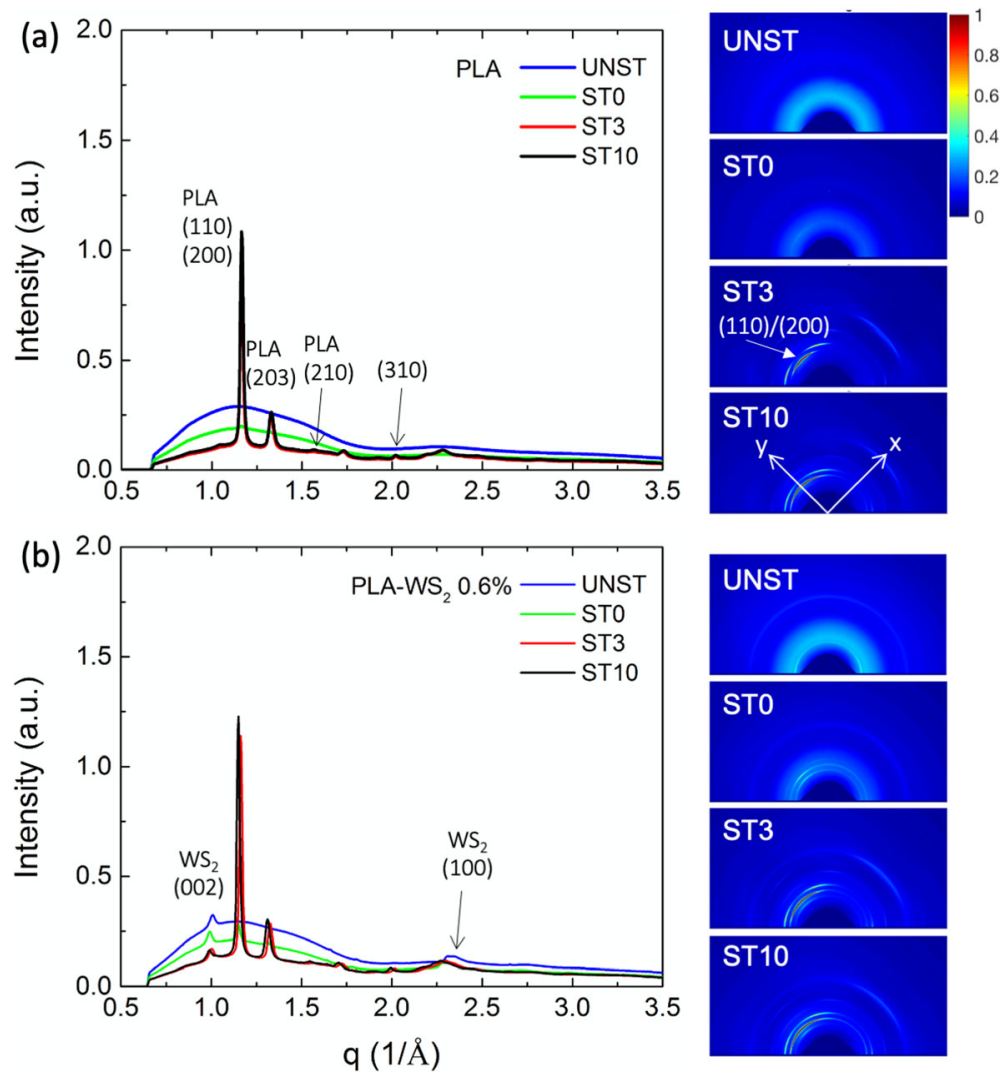

Fig. 4 WAXS $1 D$ Intensity vs. scattering vector $q$ (left) of PLA (a) and PLA-WS $20.6 \%$ (b) films obtained after integration of the corresponding 2D patterns (on the right) over the azimuth angle: UNST, STO, ST3 and ST10 films (for each type of sample only the pattern that best reproduces the average behaviour among the different measurements was selected). The intensity scale of the 2D patterns is the same for all the samples. The stretching direction is along the $x$-axis 


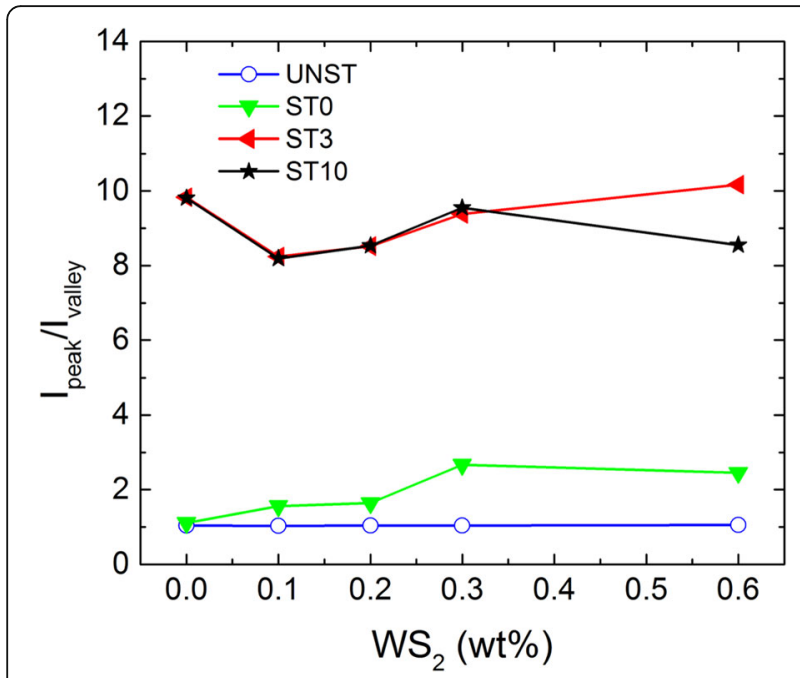

Fig. 5 Ratio of the intensity value of the main PLA reflection ((110)/ (200), centered at $q=1.16 \AA^{-1}$ ) and the intensity value at the valley (positioned at $q=1.25 \AA^{-1}$ ) $\left.\right|_{\text {peak }} / l_{\text {valley }}$ for the UNST, STO, ST3 and ST10 films as a function of the WS 2 NTs concentration ( $w$ t $\%=0$ corresponds to neat PLA)

range $250-450 \mathrm{~cm}^{-1}$ includes two weak bands of PLA (Fig. 6a); these two bands are present in the PLA-WS $0.6 \%$ spectrum (Fig. 6c) and partially overlap with two strong $\mathrm{WS}_{2}$ bands detected at 354 and $420 \mathrm{~cm}^{-1}$ (attributed to $E_{2 g}$ and $A_{1 g} \mathrm{WS}_{2}$ modes, respectively [34]). Their position is in accordance with literature data on $\mathrm{WS}_{2}$

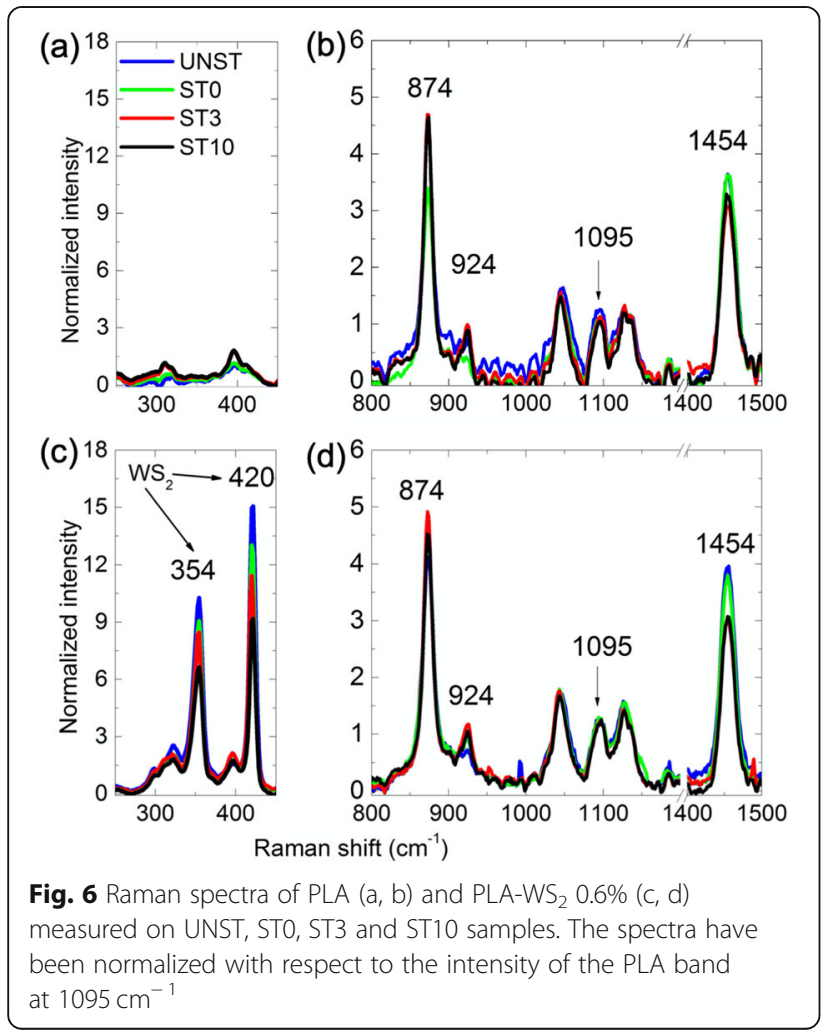

[34] and no peak shift is observed either for the presence of the PLA or upon stretching and annealing process conditions. This suggests the absence of chemical interactions between the filler and the polymer matrix and no influence of the processing conditions on the $\mathrm{WS}_{2}$ structure. As expected, the intensity of the $\mathrm{WS}_{2}$ bands increases with the $\mathrm{WS}_{2}$ concentration. This result is confirmed by the quantitative analysis of signal intensities estimated through the fitting procedure explained in the experimental section and shown in Fig. S11.

The second part of the Raman spectra (PLA, Fig. 6b and PLA- $\mathrm{WS}_{2}$ 0.6\%, Fig. 6d) shows PLA bands in the range $700-1500 \mathrm{~cm}^{-1}$ (where no band from $\mathrm{WS}_{2}$ is present). We have explicitly marked the position of four bands (centered at wavenumbers 874, 924, 1095, 1454 $\mathrm{cm}^{-1}$ ) on which we have focused the following discussion because some of these bands can be associated to the presence of crystallinity in the polymer [35-38]. Moreover, the band centered at $924 \mathrm{~cm}^{-1}$ is observed only when PLA crystallizes in $\alpha[36,38]$ and $\alpha^{\prime}[38]$ forms. These forms, indeed, present similar chain conformation $\left(10_{3}\right)$ but $\alpha^{\prime}$ is characterized by uniform conformational disorder [16]. In Table 1 we list the attributions of these bands to specific vibrations as reported in literature.

The normalized intensity of the bands (as evaluated from the fitting procedure shown in Fig. S11) centered at $924 \mathrm{~cm}^{-1}$ and $1454 \mathrm{~cm}^{-1}$ are reported as a function of $\mathrm{WS}_{2}$ concentration in Fig. $7 \mathrm{a}$ and Fig. $7 \mathrm{~b}$, respectively. As expected for amorphous samples [36], all UNST samples present negligible values of the normalized intensity of the $924 \mathrm{~cm}^{-1}$ crystalline band, while the behavior of the stretched series ST0, ST3, and ST10 is more complex. While the normalized intensity of the $924 \mathrm{~cm}^{-1}$ band for PLA ST0 is similar to that of the PLA UNST (Fig. 7a), it slightly increases for the ST0 series for the nanocomposites with $\mathrm{WS}_{2}$ up to $0.2 \mathrm{wt} \%$ and then increases more significantly above $0.2 \mathrm{wt} \%$. This result indicates, first of all, the presence of chains in conformation $10_{3}$ [36], typical of $\alpha$ and $\alpha$ forms [39] in the ST0 series; hence some level of crystallization is induced by the stretching process without further annealing. Moreover, the increase of the Raman signal of the $924 \mathrm{~cm}^{-1}$ band with the nanotube concentration confirms the nucleation activity of $\mathrm{WS}_{2}$ NTs [21-25] towards the increase of crystallinity of PLA that makes the stretching process more effective. The Raman intensity of the $924 \mathrm{~cm}^{-1}$ band for the series ST3 and ST10 is higher than the ST0 series and independent on the $\mathrm{WS}_{2}$ concentration, suggesting that prolonged annealing (above $3 \mathrm{~min}$ ) does not promote further crystallization.

A similar behavior was observed for the band at 874 $\mathrm{cm}^{-1}$ as shown in Fig. S12a even though the effects are less pronounced than at the $924 \mathrm{~cm}^{-1}$ band because the 
Table 1 Assignments of the analyzed bands (labelled in Fig. 6b) to specific vibrations of PLA chains

\begin{tabular}{llll}
\hline Wavenumber $\left(\mathbf{c m}^{-\mathbf{1}}\right)$ & Vibration mode & Associated to phase & Ref. \\
\hline 874 & $\mathrm{C}-\mathrm{COO}$ stretching & Crystalline and amorphous & [39] \\
924 & $\mathrm{CH}_{3}$ rocking and C-C bending & Crystalline & {$[39]$} \\
1095 & OCO stretching & - & {$[26]$} \\
1454 & asymmetric deformation modes of the $\mathrm{CH}_{3}$ group & - & {$[39]$} \\
\hline
\end{tabular}

$874 \mathrm{~cm}^{-1}$ band is also sensitive to the amorphous PLA phase [36].

The last band that we analyzed was the one located at wavenumber $1454 \mathrm{~cm}^{-1}$ (Fig. 7b) where the intensity values are reversed with respect to the case of the 924 $\mathrm{cm}^{-1}$ and $874 \mathrm{~cm}^{-1}$ bands: the highest intensity is observed for the UNST series, followed by the ST0 and lastly by the ST3 and ST10 series which are very similar. This trend suggests that the $1454 \mathrm{~cm}^{-1}$ band could be attributed to the amorphous content of PLA, but no other evidence has been found in literature to support this hypothesis. Further measurements at different points of the samples (we reiterate here that we only collected data in the central region of the films) may increase the statistics and reduce the magnitude of the error bar in Fig. 7b.

Regarding the position of the investigated bands, no significant Raman shift is observed as an effect of either $\mathrm{WS}_{2}$ introduction or process conditions. This suggests that there are no interactions between the filler and polymer matrix that change the force constant of the bonds in vibration, or changes in the packing of the polymer chains in the crystal. Additionally, the FWHM of the investigated $924 \mathrm{~cm}^{-1}$ band does not change with $\mathrm{WS}_{2}$ wt\%, suggesting that the crystalline domains in PLA and PLA- $\mathrm{WS}_{2}$ nanocomposites samples are very similar in terms of size and defects. (Fig. S13a).

The thermal behaviour of the PLA and PLA-WS ${ }_{2}$ samples was studied by DSC analysis, for additional information about the amorphous phase of PLA (presence of enthalpic relaxation, possible variation of $\mathrm{T}_{\mathrm{g}}$, ability to cold crystallization) as a function of the drawing and annealing processes, and of $\mathrm{WS}_{2}$ concentration. For this purpose, we focused our attention on the thermal transitions present in the first heating scan of the samples.

The thermograms of PLA and PLA- $\mathrm{WS}_{2} 0.6 \mathrm{wt} \%$ samples are compared in Fig. $8 \mathrm{a}$ and b; the temperatures and enthalpies associated to the principal thermophysical transitions are reported as a function of the $\mathrm{WS}_{2}$ amount in Fig. 8c and d, respectively.

In the heating scans of both UNST PLA and PLA$\mathrm{WS}_{2} 0.6 \mathrm{wt} \%$ samples, the thermal transitions of the polymer are observed: an endothermic peak at about $70{ }^{\circ} \mathrm{C}$, a cold crystallization exotherm between $100{ }^{\circ} \mathrm{C}$ and $130{ }^{\circ} \mathrm{C}$, and a double peak melting endotherm between $155^{\circ} \mathrm{C}$ and $175^{\circ} \mathrm{C}$.

The first endothermic peak at $70^{\circ} \mathrm{C}$ overlaps with the expected glass transition interval for PLA and is often associated to different endothermic transitions of the PLA amorphous phase (melting of mesophase [40-42], simultaneous devitrification and enthalpic relaxation of the rigid amorphous fraction [43], and physical aging [44]). Therefore, this peak is generally connected to the relaxation of the intermediate phase with some molecular ordering and it is more pronounced in samples characterized by a high percentage of the amorphous phase [26]. Indeed, the highest intensity of this peak is observed in UNST samples both in PLA and nanocomposites, that are found to be amorphous from WAXS (Figs. 4 and 5) and Raman spectroscopy (Figs. 6 and 7).
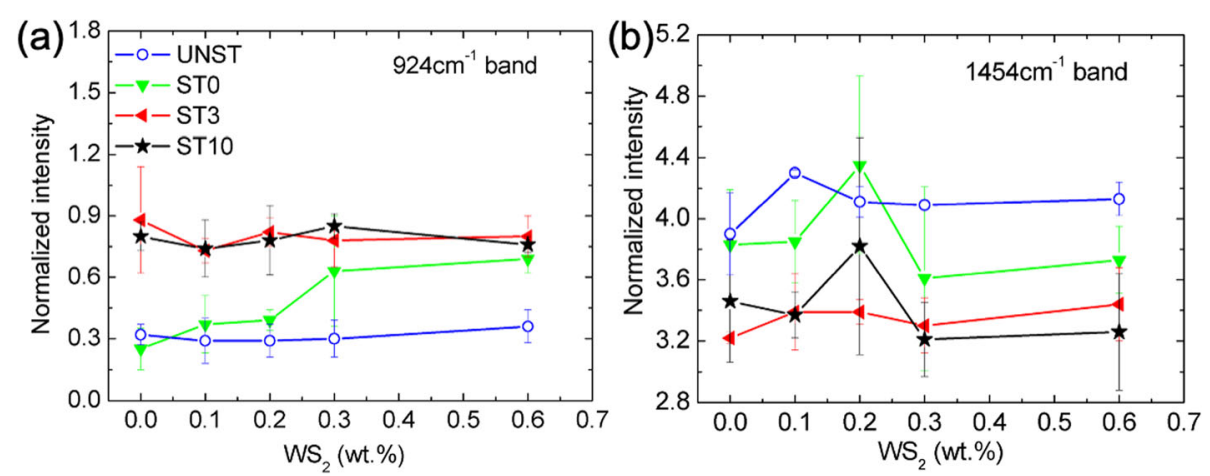

Fig. 7 Normalized Raman intensity of $924 \mathrm{~cm}^{-1}$ and $1454 \mathrm{~cm}^{-1}$ PLA bands measured in the spectra of UNST, STO, ST3, ST10 films of PLA and PLA-WS 2 nanocomposites. The data are normalized with respect to the intensity of the PLA band at $1095 \mathrm{~cm}^{-1}$ 


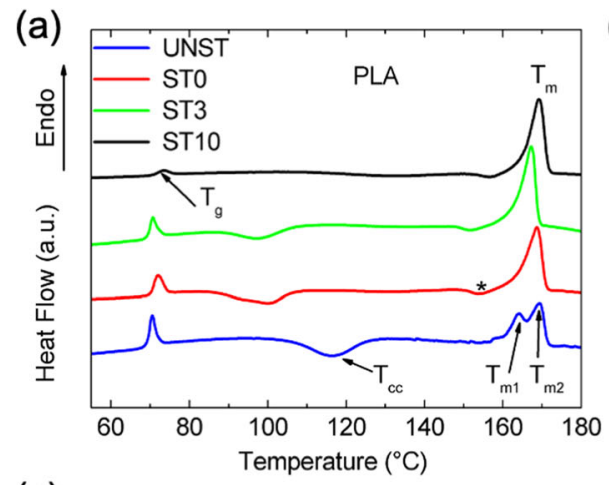

(c)

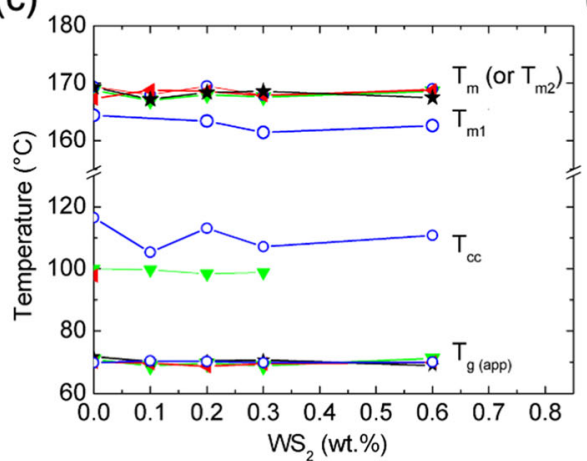

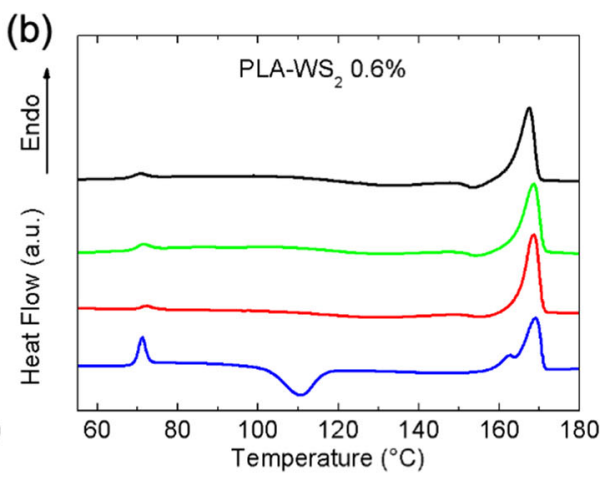

(d)

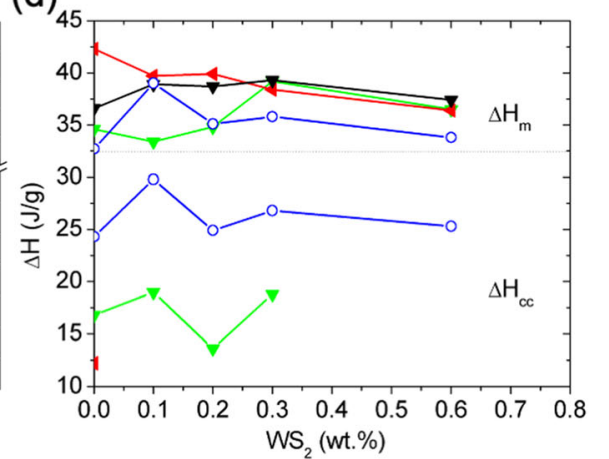

Fig. 8 Results of DSC analysis carried out by heating at $5^{\circ} \mathrm{C} / \mathrm{min}$ rate UNST, STO, ST3, ST10 films of neat PLA (a) and PLA- WS 2 0.6\% films (b); temperatures (c) and enthalpies (d) measured for the relevant phase transitions observed in the DSC thermograms of the samples

This first endothermic peak becomes less intense with stretching (ST0) and post-stretching annealing (ST3 and ST10) (Fig. 8a, b) due to the reduction of the amorphous phase by strain-induced crystallization [26]. Similarly, the enthalpy relaxation becomes less pronounced by increasing the $\mathrm{WS}_{2}$ concentration: as $\mathrm{WS}_{2}$ nanotubes favor polymer crystallization, the amount of amorphous phase (responsible for the enthalpic relaxation) decreases with increasing $\mathrm{WS}_{2}$ concentration.

Due to the overlap with enthalpic relaxation, we could make only an approximate estimation of the values of the glass transition temperature, hence we refer to it as "apparent" - $\mathrm{T}_{\mathrm{g}(\mathrm{app})}$ (measured at the inflection point of the rising edge of the endothermic peak). $T_{g(a p p)}$ remains almost constant for all the samples independently of the amount of $\mathrm{WS}_{2}$ and of the process (see Fig. 8c) suggesting that the filler introduction into polymer matrix has no measurable stiffening effect on the amorphous phase. This result is not surprising, given the low quantity of $\mathrm{WS}_{2}$ used. By continuing to heat the UNST films of PLA and PLA- $\mathrm{WS}_{2}$, an exothermic peak is observed in the range $100{ }^{\circ}-130^{\circ} \mathrm{C}$ where the cold crystallization of the amorphous phase takes place (Fig. 8a, b). The cold crystallization temperature $T_{c c}$ is plotted as a function of $\mathrm{WS}_{2}$ concentration in Fig. 8c; for the UNST samples, it slightly decreases with the concentration of $\mathrm{WS}_{2}$, in good agreement with the literature [23]. The incorporation of $\mathrm{WS}_{2}$ NTs into the PLA influences the formation of the nuclei during crystallization of PLA by decreasing the free energy of nucleation [23]. Among the ST series, the cold crystallization is detected only for the ST0 and for the ST3 samples until a $\mathrm{WS}_{2}$ concentration of $0.3 \mathrm{wt} \%$. It is absent for ST3 of PLA-WS $\mathrm{WS}_{2} 0.6 \%$ and in all ST10 of PLA- $\mathrm{WS}_{2}$, even though a melting peak is detected in the thermograms of all the ST samples. This suggests the presence of pre-existing crystals from processing according to WAXS and Raman data.

The presence or absence of cold crystallization in the heating scans of ST samples is associated with the ability of the stretching and annealing process to promote the formation of crystals due to chain orientation and changes in the amount of $\mathrm{WS}_{2}$ NTs. When the thermomechanical process is effective, the fraction of polymer chains in the amorphous state decreases. As a consequence, the cold crystallization is reduced or even suppressed [26] as in the case of all ST10 samples and for PLA-WS ${ }_{2}$ ST3 with wt\% higher than $0.3 \%$ (no $T_{c c}$ measured, Fig. 8c). For these latter samples the major role towards crystallization is played by strain and not by annealing.

As a further consideration of Fig. 8c, the value of $T_{c c}$ decreases from the UNST to the ST series, as a consequence of the stretching and of the annealing [45]. 
For the ST series, a second weak exothermic peak (highlighted with a star in Fig. 8a), centered around $155^{\circ} \mathrm{C}$, is often visible before the melting peaks, due to further crystallization occurring as the heating proceeds. This peak is characteristic of presence of PLA crystallites in $\alpha$ ' phase which change into $\alpha$ phase during the heating scan by solid-solid transition. Indeed, the more disordered $\alpha^{\prime}$ structure, generally obtained at lower temperatures than the $\alpha$ form (below $120^{\circ} \mathrm{C}$ ), is characterized by lower thermal stability than $\alpha$ form [16] and can be converted into $\alpha$ by annealing at high temperature [38].

The range of temperatures between $160^{\circ} \mathrm{C}$ and $180^{\circ} \mathrm{C}$ is where the melting of the crystals takes place (Fig. 8a, b). While a single melting peak (corresponding to a single melting temperature $T_{m}$ ) is visible for the ST samples, a double peak (corresponding to two melting temperatures $T_{m 1}$ and $T_{m 2}$ with $T_{m 1}<T_{m 2}$ ) is detected for UNST samples. According to similar observations in melt-processed PLA-WS $\mathrm{WS}_{2}$ nanocomposites, the first endothermic peak $\left(T_{m 1}\right)$ is due to the fusion of smaller and metastable crystals formed during the DSC heating process itself (giving the slow heating rate of $5{ }^{\circ} \mathrm{C} / \mathrm{min}$ ). Such crystals recrystallize at higher temperature leading to the second melting peak $\left(T_{m 2}\right)[22,23]$. The absence of a double peak due to a process of melt-crystallization in ST samples suggests that the stretching process favors the formation of more perfect and stable crystals independently of the presence of the nanotubes. The values of $T_{m 1}, T_{m 2}, T_{m}$ and $\Delta H_{m}$ are similar for all the samples (Fig. 8c, d) and are not influenced by the $\mathrm{WS}_{2}$ concentration, in good agreement with data reported in [22, 24]. As the results of Raman experiments also point out, this is a further indication that the size and degree of perfection of crystallites of PLA in PLA-WS $\mathrm{W}_{2}$ composites is similar for all the stretched samples.

The degree of crystallinity $\left(X_{c}\right)$ was evaluated from the DSC thermograms using eq. (1) in the Methods section (Table 2). For PLA (wt $\%=0)$ and PLA- $\mathrm{WS}_{2}$ with $\mathrm{WS}_{2}$ NTs concentration up to $0.3 \%$ the $X_{c}$ doubles for the effect of annealing (see values for ST0 vs. UNST) and increases four times after $3 \mathrm{~min}$ of annealing (ST3) reaching a value of about $40 \%$; a longer annealing does not further modify the $X_{c}$ (ST10 similar to ST3). For the PLA- $-\mathrm{WS}_{2} \quad 0.6 \%$ the stretching itself is enough for the sample to reach the maximum crystallinity.

The data of Table 2 are displayed in Fig. 9 and correlated with the Raman data, specifically with the ratio between the intensity of the $924 \mathrm{~cm}^{-1}$ and $1454 \mathrm{~cm}^{-1}$ bands $\mathrm{I}_{924} / \mathrm{I}_{1454}$ as an index of the amount of the crystalline phase with respect to the amorphous one as measured by Raman spectroscopy. As expected, the lowest values of $X_{c}$ and of the ratio $\mathrm{I}_{924} / \mathrm{I}_{1454}$ are measured for the UNST samples that show an amorphous structure
Table 2 Percentage of crystallinity $\left(X_{c}\right)$ evaluated from DSC data (Fig. 8) for PLA and PLA-WS 2 samples

\begin{tabular}{lllll}
\hline $\begin{array}{l}\mathbf{W S}_{\mathbf{2}} \begin{array}{l}\text { concentration } \\
\text { (wt\%) }\end{array} \\
\text { wt\% }\end{array}$ & $\boldsymbol{X}_{\boldsymbol{c}}(\%)$ & & & \\
\hline 0 & UNST & ST0 & ST3 & ST10 \\
0.1 & 9.0 & 19 & 32.1 & 38.0 \\
0.2 & 9.8 & 15.4 & 42.4 & 41.6 \\
0.3 & 10.9 & 22.7 & 42.7 & 41.4 \\
0.6 & 9.6 & 21.8 & 41.1 & 42.1 \\
\hline
\end{tabular}

from WAXS (Figs. 4 and 5). Both the stretching process and inclusion of $\mathrm{WS}_{2}$ cause an increase of $X_{c}$ and $\mathrm{I}_{924} /$ $\mathrm{I}_{1454}$. A further increment of $X_{c}$ and $\mathrm{I}_{924} / \mathrm{I}_{1454}$ after the annealing is detected for PLA and PLA-WS ${ }_{2}$ nanocomposites with $\mathrm{WS}_{2}$ wt\% up to $0.3 \%$, whereas the stretching itself suffices to induce the highest $X_{c}$ and $\mathrm{I}_{924} / \mathrm{I}_{1454}$ values in the PLA- $\mathrm{WS}_{2} 0.6 \%$.

\section{Conclusions}

We studied the crystallinity and the thermal behaviour of stretched and annealed nanocomposite films of PLA and PLA- $\mathrm{WS}_{2}$ containing $\mathrm{WS}_{2}$ nanotubes at concentrations from $0.1 \%$ to $0.6 \%$ by weight. The samples were prepared by stretching amorphous films up to $100 \%$ strain held at $90^{\circ} \mathrm{C}$ and annealed at the same temperature for different times: 0,3 and $10 \mathrm{~min}$. WAXS, Raman spectroscopy and DSC analyses showed that increasing the nanotube concentration enhances the crystallinity of PLA induced by the stretching process. In particular, WAXS and Raman data proved that a $\mathrm{WS}_{2}$ NTs concentration of $0.3 \mathrm{wt} \%$ leads to more than twofold increase of crystallinity with respect to neat, stretched PLA. The effect of the additional annealing step on the crystallinity is more evident for samples with $\mathrm{WS}_{2}$ content lower than the maximum concentration

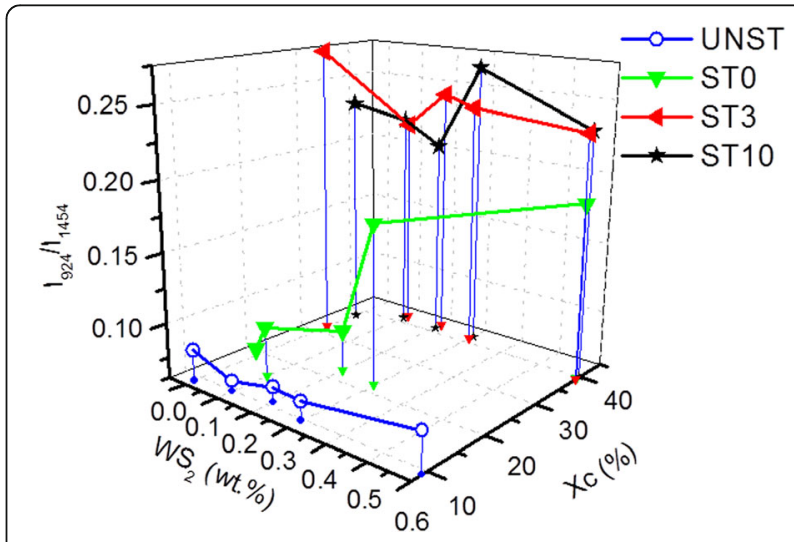

Fig. 9 Degree of crystallinity $X_{c}$ calculated from DSC data and intensity ratio $I_{924} / I_{1454}$ of Raman bands as function of wt $\%$ of $W_{2}$ for ST and UNST samples of PLA and PLA-WS 2 nanocomposites 
examined $(0.6 \mathrm{wt} \%)$ here. At this concentration, the presence of $\mathrm{WS}_{2}$ NTs allows the stretching process to dominate, enabling comparable levels of crystallinity even at lower annealing times. The results discussed in the present work may impact the choice of processing conditions of a novel class of PLA-WS $\mathrm{W}_{2}$ nanocomposites for multiple applications.

\section{Supplementary Information}

The online version contains supplementary material available at https://doi. org/10.1186/s42252-021-00016-2.

\section{Additional file 1. \\ Additional file 2. \\ Additional file 3. \\ Additional file 4. \\ Additional file 5 . \\ Additional file 6 . \\ Additional file 7. \\ Additional file 8. \\ Additional file 9. \\ Additional file 10. \\ Additional file 11. \\ Additional file 12 \\ Additional file 13.}

\section{Abbreviations}

NTs: Nanotubes; WS 2 : Tungsten Disulfide; PLA: Poly(lactic acid); PLAWS 2 : Nanocomposites of PLA and WS 2 ; WAXS: Wide Angle X-Ray Scattering; DSC: Differential Scanning Calorimetry; BVSs: Bioresorbable Vascular Scaffolds; wt\%: Weight percent; UNST: Unstretched; ST: Stretched at T $=90^{\circ} \mathrm{C}$; STO: Stretched at T $=90^{\circ} \mathrm{C}$ but not annealed; ST3: Stretched at T $=90^{\circ} \mathrm{C}$ and annealed for 3 min at the same temperature; FWHM: Full Width at Half Maximum; $T_{g}$ : Glass transition; $T_{m}$ : Melting temperature corresponding with the maximum temperature position measured in a single melting process; $\mathrm{T}_{\mathrm{m} 1}$ : Melting temperature measured in a double melting peak and corresponding to the first melting peak observed in the endothermic process.; $T_{m 2}$ : Melting temperature measured in a double melting peak and corresponding to the second melting peak observed; CC: Cold-crystallization: $T_{c c}$ : Cold-crystallization temperature corresponding with the minimum temperature position measured in the exothermic process; $\Delta \mathrm{H}_{c c}$ : Coldcrystallization enthalpy; $\Delta H_{m}$ : Melting enthalpy; $X_{c}$ : Crystalline grade obtained by DSC measurements after stretching and annealing process

\section{Acknowledgments}

The authors kindly acknowledge the Department of Industrial Engineering, (Prof. Giuliana Gorrasi), University of Salerno, Italy for the support in the tensile tests. Steven Weigand, beamline scientist at Beamline 5-ID-D of the APS at ANL, is especially acknowledged for his support with WAXS data collection and analysis. Dr. Alla Zak (Holon Institute of Technology) is acknowledged for providing a special purchase price of the WS 2 NTs from NanoMaterials in 2011 and useful discussions on their purification and dispersion.

\section{Authors' contributions}

Di Luccio and Kornfield designed the research, Di Luccio coordinated the activities. Tammaro manufactured the samples. Tammaro, Borriello, Villani, Loffredo, Ramachandran and Di Luccio performed experiments and analyzed the data. De Vito developed the code for Raman data analysis. All the authors reviewed and approved the manuscript.

\section{Funding}

This project has received funding from the European Union's Horizon 2020 research and innovation programme under the Marie Skłodowska-Curie grant agreement No 691238, the Jacobs Institute for Molecular Engineering for Medicine at the California Institute of Technology, and the National Heart, Lung, And Blood Institute of the National Institutes of Health under Award Number F31HL137308.

\section{Availability of data and materials}

Not applicable.

\section{Competing interests}

The authors declare that they have no competing interests.

\section{Author details}

1Division for Sustainable Materials, ENEA - Italian National Agency for New Technologies, Energy and Sustainable Economic Development, Piazzale E. Fermi 1, 80055 Portici, NA, Italy. ${ }^{2}$ Division of Chemistry and Chemical Engineering, California Institute of Technology, Pasadena, CA 91125, USA. ${ }^{3}$ Division for Photovoltaic and Smart Devices, ENEA - Italian National Agency for New Technologies, Energy and Sustainable Economic Development, Piazzale E. Fermi 1, 80055 Portici, NA, Italy.

Received: 23 October 2020 Accepted: 3 January 2021

Published online: 26 January 2021

\section{References}

1. M. Jamshidian, E.A. Tehrany, M. Imran, M. Jacquot, S. Desobry, Poly(lactic acid): Production, applications, nanocomposites, and release studies. Compr. Rev. Food Sci. Food Saf. 9, 552-571 (2010)

2. H. Tsuji, Autocatalytic hydrolysis of amorphous-made polylactides: Effects of I-lactide content, tacticity, and enantiomeric polymer blending. Polymer 43(6), 1789-1796 (2002)

3. H. Tsuji, In vitro hydrolysis of blends from enantiomeric poly(lactide)s. part 4: Well-homo-crystallized blend and nonblended films. Biomaterials 24(4), 537-547 (2003)

4. D.W. Grijpma, H. Altpeter, M.J. Bevis, J. Feijen, Improvement of the mechanical properties of poly(D, L-lactide) by orientation. Polym. Int. 51(10), 845-851 (2002)

5. S. Zhou, X. Deng, X. Li, W. Jia, L. Liu, Synthesis and characterization of biodegradable low molecular weight aliphatic polyesters and their use in protein-delivery systems. J. Appl. Polym. Sci. 91(3), 1848-1856 (2004)

6. S. Jacobsen, H.-G. Fritz, Plasticizing polylactide-The effect of different plasticizers on the mechanical properties. Polym. Eng. Sci. 39(7), 1303-1310 (1999)

7. H. Wang, Z. Qiu, Crystallization kinetics and morphology of biodegradable poly(L-lactic acid)/graphene oxide nanocomposites: Influences of graphene oxide loading and crystallization temperature. Thermochim. Acta 527, 40-46 (2012)

8. D. Cohn, A. Hotovely Salomon, Designing biodegradable multiblock PCL/ PLA thermoplastic elastomers. Biomaterials 26(15), 2297-2305 (2005)

9. M. Day, A.V. Nawaby, X. Liao, A DSC study of the crystallization behavior of polylactic acid and its nanocomposites. J. Therm. Anal. Calorim. 86(3), 623629 (2006)

10. P. Pan, Z. Liang, A. Cao, Y. Inoue, Layered metal phosphonate reinforced poly (L-lactide) composites with a highly enhanced crystallization rate. ACS Appl. Mater. Interfaces 1(2), 402-411 (2009)

11. G. Papageorgiou, D. Achilias, S. Nanaki, T. Beslikas, D. Bikiaris, PLA nanocomposites: Effect of filler type on non-isothermal crystallization. Thermochim. Acta 511(1), 129-139 (2010)

12. Y. Zhao, Z. Qiu, W. Yang, Effect of functionalization of multiwalled nanotubes on the crystallization and hydrolytic degradation of biodegradable poly (L-lactide). J. Phys. Chem. B 112(51), 16461-16468 (2008)

13. H. Pan, Z. Qiu, Biodegradable poly (L-lactide)/polyhedral oligomeric silsesquioxanes nanocomposites: Enhanced crystallization, mechanical properties, and hydrolytic degradation. Macromolecules 43(3), 1499-1506 (2010)

14. J. Yu, Z. Qiu, Preparation and properties of biodegradable poly(L-lactide)/ octamethyl-polyhedral oligomeric silsesquioxanes nanocomposites with 
enhanced crystallization rate via simple melt compounding. ACS Appl. Mater. Interfaces 3(3), 890-897 (2011)

15. W.S. Chow, S.K. Lok, Thermal properties of poly(lactic acid)/ organomontmorillonite nanocomposites. J. Therm. Anal. Calorim. 95(2), 627-632 (2009)

16. J. Shi, X. Lu, H. Li, D. Li, Isothermal crystallization kinetics and melting behavior of PLLA/f-MWNTs composites. Therm. Anal. Calorim. 117, 13851396 (2014). https://doi.org/10.1007/s10973-014-3885-1

17. I. Kaplan-Ashiri, S.R. Cohen, K. Gartsman, V. Ivanovskaya, T. Heine, G. Seifert, I. Wiesel, H.D. Wagner, R. Tenne, On the mechanical behavior of $\mathrm{WS}_{2}$ nanotubes under axial tension and compression. Proc. Natl. Acad. Sci. U. S. A. $103,523-528$ (2006)

18. K. Ramachandran, Ph.D. thesis "Bioresorbablee Vascular Scaffolds Gain Ductility, Resistance to Hydrolysis, and Radial Strength via a Unique Poly Llactide Microstructure", (2019) doi:https://doi.org/10.7907/9146-5159. https:// resolver.caltech.edu/CaltechTHESIS:11162018-140640407

19. M. Pardo, T. Shuster-Meiseles, S. Levin-Zaidman, A. Rudich, Y. Rudich, Low cytotoxicity of inorganic nanotubes and fullerene-like nanostructures in human bronchial epithelial cells: Relation to inflammatory gene induction and antioxidant response. Environ. Sci. Technol. 48, 3457-3466 (2014)

20. E.B. Goldman, A. Zak, R. Tenne, E. Kartvelishvily, S. Levin-Zaidman, Y. Neumann, R. Stiubea-Cohen, A. Palmon, A.H. Hovav, D.J. Aframian, Biocompatibility of tungsten disulfide inorganic nanotubes and fullerenelike nanoparticles with salivary gland cells. Tissue Eng. Part A 21, 1013-1023 (2015)

21. M. Naffakh, C. Marco, G. Ellis, Development of novel melt-processable biopolymer nanocomposites based on poly(L-lactic acid) and WS 2 inorganic nanotubes. Cryst. Eng. Comm 16, 5062-5072 (2014)

22. M. Naffakh, C. Marco, G. Ellis, Non-isothermal cold-crystallization behavior and kinetics of poly(L-lactic acid)/WS 2 inorganic nanotube nanocomposites. Polymers 7, 2175-2189 (2015). https://doi.org/10.3390/polym7111507

23. M. Naffakh, C. Marco, Isothermal crystallization kinetics and melting behavior of poly (L-lactic acid) $/ \mathrm{WS}_{2}$ inorganic nanotube nanocomposites. J. Mater. Sci. 50, 6066-6074 (2015). https://doi.org/10.1007/s10853-015-9156-0

24. H. Shalom, X. Sui, O. Elianov, V. Brumfeld, R. Rosentsveig, I. Pinkas, Y. Feldman, N. Kampf, H. Wagner, N. Lachman, R. Tenne, Nanocomposite of poly(L-lactic acid) with inorganic nanotubes of WS 2 . Lubricants 7, 28-1-2821 (2019)

25. L. Tammaro, T. Di Luccio, C. Borriello, F. Loffredo, K. Ramachandran, F. Villani, F. Di Benedetto, T. Schiller, C. Minarini, J.A. Kornfield, Effect of tungsten disulfide $\left(\mathrm{WS}_{2}\right)$ nanotubes on structural, morphological and mechanical properties of poly(L-Lactide) (PLLA) films. AIP Conference Proc. 1981, 020073-1-020073-4 (2018). https://doi.org/10.1063/1.5045935

26. K. Bruckmoser, K. Resch, Effect of processing conditions on crystallization behavior and mechanical properties of poly(lactic acid) staple fibers. J. Appl. Polym. Sci. 132(33), 42432-1-42432-10 (2015). https://doi.org/10.1002/APP. 42432

27. E.W. Fischer, H.J. Sterzel, G. Wegner, Investigation of the structure of solution grown crystals of lactide copolymers by means of chemical reactions. Kolloid Z. Z. Polym. 251, 980-990 (1973)

28. S. Farah, D.G. Anderson, R. Langer, Physical and mechanical properties of PLA, and their functions in widespread applications - A comprehensive review. Adv. Drug Deliv. Rev. 107, 367-392 (2016)

29. F. Rezgui, M. Swistek, J.M. Hiver, C. G'Sell, T. Sadoun, Deformation and damage upon stretching of degradable polymers (PLA and PCL). Polymer 46(18), 7370 (2005). https://doi.org/10.1016/j.polymer.2005.03.116

30. M. Baiardo, G. Frisoni, M. Scandola, M. Rimelen, D. Lips, K. Ruffieux, E. Wintermantel, Thermal and mechanical properties of plasticized poly(L-lactic acid). J. Appl. Polym. Sci. 90, 1731-1738 (2003)

31. S.M. Mirkhalaf, M. Fagerström, The mechanical behavior of Polylactic acid (PLA) films: Fabrication, experiments and Modelling. Mech. Time-Depend. Mat. (2019). https://doi.org/10.1007/s11043-019-09429-w

32. J.S. Bergstrom, D. Hayman, An overview of mechanical properties and material modeling of Polylactide (PLA) for medical applications. Ann Biomed. Eng. 44(2), 330-340 (2016). https://doi.org/10.1007/s10439-0151455-8

33. K. Billimoria, E.L. Heeley, N. Parsons, L. Figiel, An investigation into the crystalline morphology transitions in poly-L-lactic acid (PLLA) under uniaxial deformation in the solid-state regime. Eur. Polym. J. 101, 127-139 (2018)

34. A. Berkdemir, H.R. Gutiérrez, A.R. Botello-Méndez, N. Perea-Lòpez, A.L. Elìas, C.I. Chia, B. Wang, V.H. Crespi, F. Lòpez-Urìas, J.C. Charlier, H. Terrones, M.
Terrones, Identification of individual and few layers of $\mathrm{WS}_{2}$ using Raman spectroscopy. Sci. Rep. 3, 1755-1-1755-8 (2013)

35. G. Kister, G. Cassanas, M. Vert, B. Pauvert, A. Terol, Vibrational analysis of poly(L-lactic acid). J. Raman Spectros. 26(4), 307-311 (1995)

36. G. Kister, G. Cassanas, M. Vert, Effects of morphology, conformation and configuration on the IR and Raman spectra of various poly(lactic acid)s. Polymer 39(2), 267-273 (1998)

37. K.V. Herrera, A. Misiun, A. Vogt, Preparation, and characterization of poly (lactic acid) / poly (methyl methacrylate) tablets compressed for application in quantitative analysis by Raman microspectroscopy. J. Raman Spectrosc. 46, 273-279 (2015)

38. J.P. Kalish, K. Aou, X. Yang, S. Ling Hsu, Spectroscopic and thermal analyses of $a^{\prime}$ and a crystalline forms of poly(L-lactic acid). Polymer 52, 814-821 (2011)

39. K. Wasanasuk, K. Tashiro, Crystal structure and disorder in poly(L-lactic acid) $\delta$ form ( $a^{\prime}$ form) and the phase transition mechanism to the ordered a form. Polymer 52, 6097-6109 (2011)

40. G. Stoclet, R. Seguela, J.M. Lefebvre, C. Rochas, New insights on the straininduced mesophase of poly(D,L-lactide): In situ WAXS and DSC study of the thermo-mechanical stability. Macromolecules 43, 7228-7237 (2010)

41. G. Stoclet, R. Seguela, J.M. Lefebvre, S. Elkoun, C. Vanmansart, Strain-induced molecular ordering in polylactide upon uniaxial stretching. Macromolecules 43, 1488-1498 (2010). https://doi.org/10.1021/ma9024366

42. C. Zhou, H. Li, Y. Zhang, F. Xue, S. Huang, H. Wen, J. Li, J. de Claville Christiansen, D. Yu, Z. Wu, S. Jiang, Deformation and structure evolution of glassy poly(lactic acid) below the glass transition temperature. Cryst. Eng. Comm. 17, 5651-5663 (2015)

43. Q. Lan, Y. Li, H. Chi, Highly enhanced mesophase formation in glassy poly $(\mathrm{L}-$ lactide) at low temperatures by low-pressure CO2 that provides moderately increased molecular mobility. Macromolecules 49, 2262-2271 (2016)

44. P. Pan, B. Zhu, T. Dong, K. Yazawa, T. Shimizu, M. Tansho, Y. Inoue, Conformational and microstructural characteristics of poly(L-lactide) during glass transition and physical aging. J. Chem. Phys. 129(8), 184902-1184902-10 (2008)

45. X.-R. Gao, Y. Li, H.-D. Huang, J.-Z. Xu, L. Xu, X. Ji, G.-J. Zhong, Z.M. L, Extensional stress-induced orientation and crystallization can regulate the balance of toughness and stiffness of polylactide films: Interplay of oriented amorphous chains and crystallites. Macromol 52, 5278-5288 (2019)

\section{Publisher's Note}

Springer Nature remains neutral with regard to jurisdictional claims in published maps and institutional affiliations.

\section{Submit your manuscript to a SpringerOpen ${ }^{\circ}$ journal and benefit from:}

- Convenient online submission

- Rigorous peer review

- Open access: articles freely available online

High visibility within the field

- Retaining the copyright to your article

Submit your next manuscript at $>$ springeropen.com 